ఠ

\title{
Role of Shwachman-Bodian-Diamond syndrome protein in translation machinery and cell chemotaxis: a comparative genomics approach
}

This article was published in the following Dove Press journal:

Advances and Applications in Bioinformatics and Chemistry

20 September 2011

Number of times this article has been viewed

\section{Olga Vasieva}

Institute of Integrative Biology, University of Liverpool, Liverpool, United Kingdom; Fellowship for the Interpretation of Genomes, Burr Ridge, IL, USA
Correspondence: Olga Vasieva Institute of Integrative Biology, University of Liverpool, Crown St, Liverpool, L69 7ZB, United Kingdom Tel +44 I5 I795 5 I40

Fax +44 I5 I795 4406

Email o.vasieva@liv.ac.uk
Abstract: Shwachman-Bodian-Diamond syndrome (SBDS) is linked to a mutation in a single gene. The SBDS proinvolved in RNA metabolism and ribosome-associated functions, but SBDS mutation is primarily linked to a defect in polymorphonuclear leukocytes unable to orient correctly in a spatial gradient of chemoattractants. Results of data mining and comparative genomic approaches undertaken in this study suggest that SBDS protein is also linked to tRNA metabolism and translation initiation. Analysis of crosstalk between translation machinery and cytoskeletal dynamics provides new insights into the cellular chemotactic defects caused by SBDS protein malfunction. The proposed functional interactions provide a new approach to exploit potential targets in the treatment and monitoring of this disease.

Keywords: Shwachman-Bodian-Diamond syndrome, wybutosine, tRNA, chemotaxis, translation, genomics, gene proximity

\section{Introduction}

Shwachman-Bodian-Diamond syndrome (SBDS) is an autosomal recessive disorder characterized by pancreatic exocrine insufficiency, bone marrow dysfunction, and skeletal abnormalities. ${ }^{1}$

The SBDS gene ( $s b d s$ ) encodes a member of a highly conserved protein family of unknown function, with orthologs in diverse species, including Archaea, plants, and eukaryotes, but not Eubacteria. ${ }^{2}$ Structural and functional aspects of SBDS link this protein to a group of proteins involved in RNA metabolism with ribosome-associated functions., ${ }^{3,4}$ For example, the yeast SBDS ortholog clusters with RNA-processing enzymes and ribosomal RNA-processing factors in gene expression analyses, 5,6 and in the SBDS sequence homolog in Saccharomyces cerevisiae, YLR022C, was shown to be physically associated with proteins involved in ribosome biosynthesis. ${ }^{7}$

The N-terminal domain of the SBDS protein, which is most prone to mutations leading to disease, contains a novel mixed $\alpha / \beta$ fold that was also suggested for a single domain yeast protein, Yhr087wp, implicated in RNA metabolism. ${ }^{1}$ The $\beta \alpha \beta \beta \alpha \beta$ folding topology of the SBDS C-terminal domain III $\left(\mathrm{Glu}^{162}-\mathrm{Gly}^{234}\right)$ is structurally close to domain $\mathrm{V}$ of $S$. cerevisiae elongation factor 2 (Eft2p/Ydr385wp). ${ }^{8}$ It is also typical for a ferredoxin-like fold and characteristic of a number of heterogeneous nuclear ribonucleoproteins, eg, factors involved in regulation of alternative splicing. ${ }^{9}$ Although SBDS links to RNA metabolism are apparent, an association has also been observed between the SBDS protein and cell locomotion, in that polymorphonuclear leukocytes isolated from SBDS patients are unable to orient correctly in a 
spatial gradient of chemoattractants. ${ }^{10}$ SBDS protein was also shown to localize to the pseudopod of Dictyostelium amoebae during chemotaxis. ${ }^{11}$ The interaction of SBDS and a structural or regulatory cytoskeletal component is more than likely responsible for the observed defect in polymorphonuclear leukocyte chemotaxis. Nonetheless, no specific candidate for such an interaction has been suggested.

The complexities of the myriad defects associated with SBDS have made it difficult to relate the diverse biochemical and phenotypic properties of the SBDS syndrome on an experimental basis. A way forward on how mutations of the SBDS gene ( $s b d s)$ may relate to its altered functions is via a functional genomics approach and phylogenetic analysis of gene proximity in $s b d s$ loci. Functionally related genes are commonly found clustered in prokaryotic and eukaryotic genomes, ${ }^{12-17}$ and predicting gene function based on physical proximity to other genes has been used successfully in a number of studies. Therefore, we treated consistency of gene proximity in $s b d s$ loci in evolutionary distant genomes as an indication of functional relatedness, which led to a prediction of SBDS protein involvement in initiation of translational wybutosine metabolism. The crosstalk between the translation machinery and elements of the cytoskeleton provides an explanation as to how cell chemotactic defects may be caused by SBDS malfunction.

\section{Materials and methods}

We used the Seed database (http://theseed.uchicago.edu/FIG/ index.cgi) for chromosome alignment and phylogenetic analysis of gene positional clusters. ${ }^{18}$ The "Compare Regions" resource provided by Seed allows alignment of chromosome loci that contain open reading frames for homologous proteins, or, in other words, to pin these loci through genes that are homologous to a query sequence. It can be used in a text or graphic format. We used the latter to illustrate phylogenetic conservation of gene proximity. The typical graphic window presents a selected number of chromosome loci from different genomes. The first line of Compare Regions is a graphical display of the chromosomal neighborhood of the features in its genome. All proteins are shown as colored arrows, where the direction depicts the strand of the feature. RNAs and other features are small boxes on the line. Feature overlaps are resolved by drawing the overlapping feature in a new line. The graph is centered on the selected feature, always numbered 1 and colored red. Below, there is the same region for orthologs in other organisms, also colored in red. The colors of the other features (as well as the numbers) also represent ortholog (or sometimes also paralog) features.
Whenever there are at least two ortholog or paralog features of a kind, a color (and a number) is assigned to them. The selection of genomes to show in the graphics can be made by similarity or the pair of close homologs pin. We used similarity, which means that the genomes are chosen using the similarity of the selected genes to its orthologs in other genomes. The $\mathrm{E}$ value cutoff for selection of pinned coding sequence depicts the minimum similarity in order for its region to be displayed. We used the e-20 E value threshold to obtain all the presented data sets.

There are numerous queering and display options that allow customization of the size of displayed regions, selection of organisms, similarity thresholds for pinning of regions, and coloring of features that we implemented to deliver the illustrations accompanying this paper.

\section{Results \\ Conservation of gene proximity in SBDS gene loci}

Phylogenetic analysis of archeal sbds loci (Figure 1) shows conservation of $s b d s$ gene proximity. $S b d s$ orthologs are shown as red arrows (N1) in the centers of all the selected regions, where one can also see repetitive occurrence of colors/numbers depicting other orthologous genes in different genomes. Almost all of these co-occurring genes are related to RNA modification and degradation, ie, probable exosome complex exonuclease 2 (EC 3.1.13.-)/tRNA nucleotidyltransferase (N2), proteasome subunit $\alpha$ (EC 3.4.25.1) (N3), probable exosome complex RNA-binding protein 1 (N4), large ribosomal subunit protein L37 Ae (N5) large ribosomal subunit protein $\mathrm{L} 15 \mathrm{e}(\mathrm{N} 7)$, ribonuclease $\mathrm{P}$ (tRNA processing) protein component 3 (EC 3.1.26.5) (N8), ribonuclease $\mathrm{P}$ protein component 2 (EC 3.1.26.5) (N9), prefoldin, chaperonin cofactor (N10), and a predicted exosome subunit containing the IMP4 domain present in small nuclear ribonucleoprotein (N11). An archeal $s b d s$ locus that includes all or part of the genes encoding the listed functions is surrounded by a variable region (gray arrows), suggesting that clustered genes related to the archeal exosome complex indeed represent a functionally coupled group or even an operon, and that $s b d s$ can be a part of this group.

Chromosome loci with vertebrate orthologs to human $s b d s$ (ENSG00000126524) have also been aligned. In the human genome, sbds spans a start for tywl (ENSG00000198874), which encodes tRNA-wybutosine synthesizing protein (TYW1) from the opposite string. In all vertebrate genomes analyzed (from the SEED database collection), sbds (red, N1) 


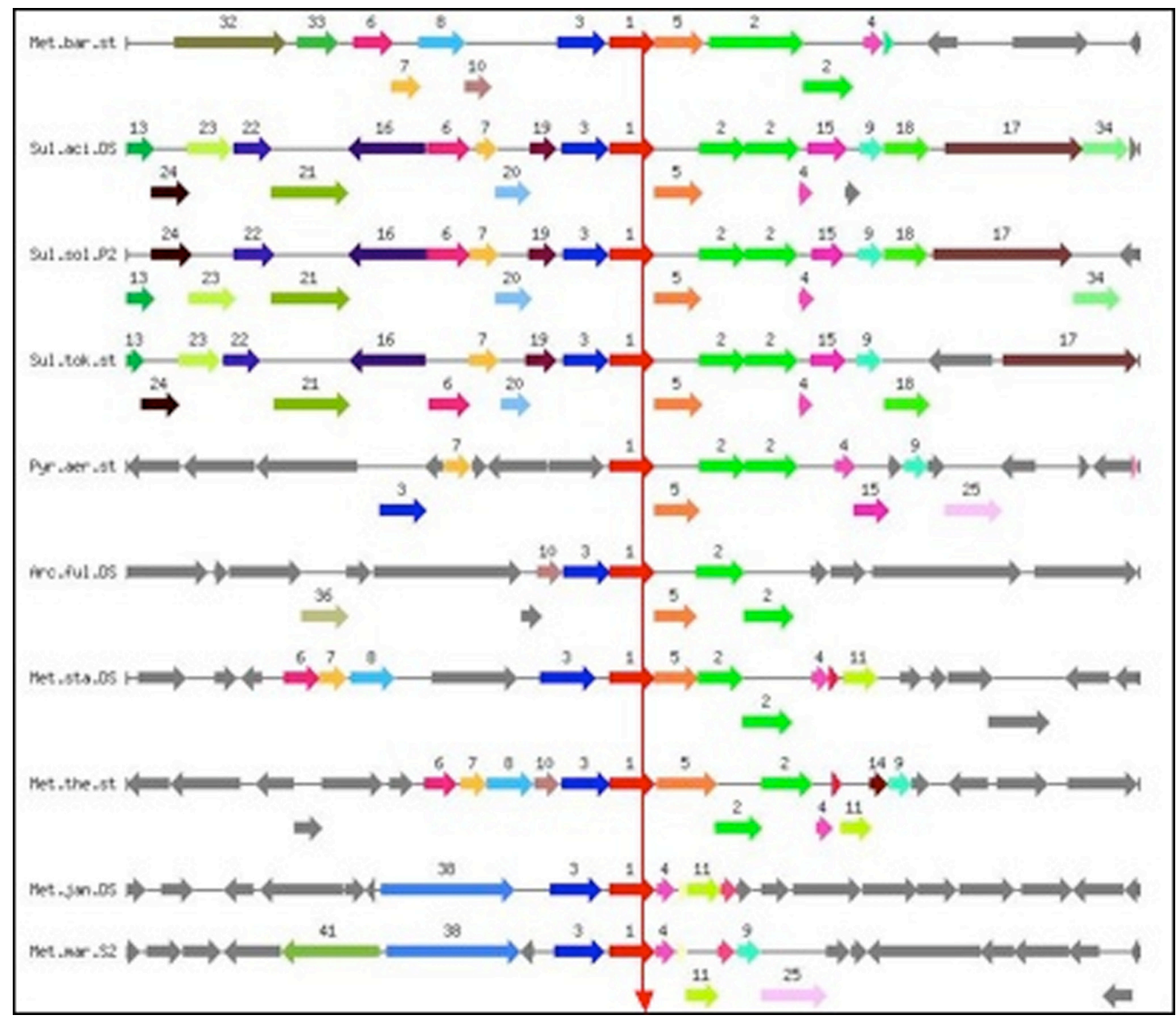

Figure I Graphical display of the chromosomal neighborhood of sbds genes in archeal genomes. Arrows correspond to open reading frames, same color and number depicts gene orthology. Vertical arrow connects pinned genes (red arrows), ie, orthologs of sbds gene from Methanosarcina barkeri (upper line). Genes encode I-SBDS protein (AAB90746.I and its orthologs), 2-probable exosome complex exonuclease 2 (EC 3.1.13.-)/tRNA nucleotidyltransferase, 3-proteasome subunit $\alpha$ (EC 3.4.25.I), 4-probable exosome complex RNA-binding protein I, 5-large ribosomal subunit protein L37 Ae, 7-large ribosomal subunit protein LI5e, 8-ribonuclease P (tRNA processing) protein component 3 (EC 3.I.26.5), 9-ribonuclease P protein component 2 (EC 3.I.26.5), I0-prefoldin, chaperonin cofactor, and I I-predicted exosome subunit containing the IMP4 domain present in small nuclear ribonucleoprotein. $16 \mathrm{kbp}$ regions are shown.

Abbreviations: Met. sta.DS, Methanosphaera stadtmanae DSM 3091; Met.the.st- Methanothermobacter thermautotrophicus; Met.mar.S, Methanococcus maripaludis S2; Arc.ful, Archaeoglobus fulgidus DSM 4304; Pyr.aer.st, Pyrobaculum aerophilum str. IM2; Met.bar.st, Methanosarcina barkeri str. Fusaro; Sul.aci.ds, Sulfolobus acidocaldarius DSM 639; Sul.sol. P2, Sulfolobus solfataricus P2; Sul.tok.st, Sulfolobus tokodaii str. 7.

is colocalized with a gene encoding the TYW1 homolog, annotated in SEED as wybutosine biosynthesis reductase (green, N2, Figure 2). A small gap between these two genes in the different vertebrate genomes suggests that they may share a regulatory region. The gene region downstream of $s b d s$ is not conserved, even in narrower phylogenetic groups, because one can see it for the displayed regions from several mammalian genomes.

In the Plasmodium falciparum and Ciona intestinalis genomes, $s b d s$ genes are also colocalized with genes encoding functions related to translational initiation and mainly with tRNA-regulated components of translational initiation complexes, ie, a gene for predicted GCN1 (general control of amino-acid synthesis 1) (XP_002120729.1) is immediately downstream and a gene for tRNA/RNA cytosine-C5-methylase (EC 2.1.1.-) is immediately upstream from the human $s b d s$ ortholog (XM_002124782.1) in the C. intestinalis genome (Table 1). The $a I F 2 \beta$ gene is the second gene upstream from the sbds ortholog (XP_001348280.1) in a Plasmodium genome (Table 2).

In Schizosaccharomyces pombe, the sbds ortholog (O14179, SDO1) is proximal to a gene encoding the L26e ribosomal protein, followed by a gene for the mRNA capping enzyme subunit. Gene encoding RNA processing factor 1 is immediately downstream, followed by one for S23e (Table 3).

No specific functional gene overrepresentation at the sbds locus in S. cereviciae was observed, apart from a noticeable but comparatively distant proximity to genes 


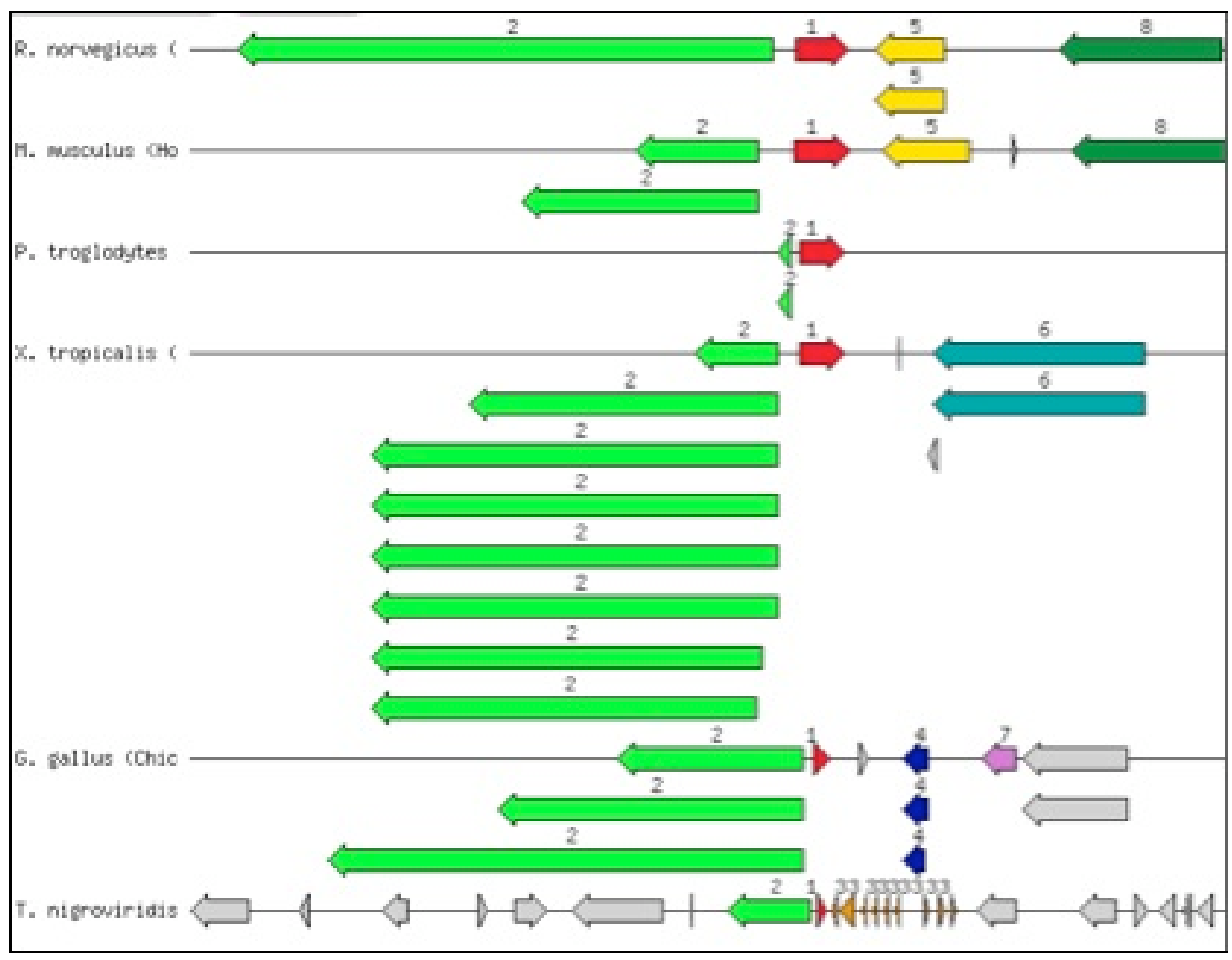

Figure 2 Graphical display of the chromosomal neighborhood of sbds genes in genomes of vertebrates. Arrows correspond to open reading frames encoding: red, ShwachmanBodian-Diamond syndrome (NM_00I008289.I and pinned orthologs, green), tRNA-YW synthesizing protein (NP_00II00607.I (in Rattus norvegicus) and ortholog, multiple arrows in Xenopus tropicalis correspond to known transcripts), 3-claudin, 4-septin, 5-06I0007 L0IRik protein, 6-hypothetical protein, 7-putative dual specificity testis-specific protein kinase 2 (EC 2.7.II.I), and 8-RABX5 (NP_00I 185988.I). 200 kbp regions are shown. Organisms are R. norvegicus, Mus musculus, Pan troglodytes, X. tropicalis, Gallus gallus, Tetraodon nigroviridis.

encoding RNA-related functions. However, $s b d s$ orthologs (XP_387070.1, XP_956795.1, XP_360288.1) are proximal to genes encoding L18e in fungal (Gibberella zeae, Neurospora crassa, and Magnaporthe oryzae) genomes (Figure 3).

\section{Discussion}

SBDS is a complex disease linked to mutation in a single gene. Recent advances in genomics and functional bioinformatics are providing a new avenue for studying this and similar phenomena, complementing traditional hypothesisdriven laboratory research. Our bioinformatics approach undertaken in this study provides a new insight as to how a single gene mutation may affect diverse molecular functions, such as RNA metabolism, translation initiation, and cytoskeletal dynamics leading to complex disease.

Phylogenetic conservation of gene proximity may manifest itself in the presence of sets of orthologous genes in gene loci from different genomes. However, even in prokaryotes, it often comes up as conservation of positional connections between and inside particular pathways, not exactly the same representatives of the pathways. ${ }^{12,18}$ Therefore, integration of genomic data may uncover trends in gene positional clustering and the underlying functional links between genes and pathways. Analysis of our data points to initiation of translation as the main functional

Table I Tabular display of the chromosomal neighborhood of sbds gene in Ciona intestinalis genome (SEED db IDs)

\begin{tabular}{llllll}
\hline ID & Start & Stop & Size $(\mathbf{n t})$ & Strand & Function \\
\hline Fig|7719.3.peg.1816 & 37845 & 18631 & 19215 & - & GCNI-like protein I \\
Fig|7719.3.peg.1817 & $\mathbf{4 6 1 4 4}$ & $\mathbf{4 5 3 8 6}$ & $\mathbf{7 5 9}$ & - & Shwachman-Bodian-Diamond syndrome protein \\
Fig|7719.3.peg.1818 & 77340 & 70503 & 6838 & - & tRNA/RNA cytosine-C5-methylase (EC 2.I.I.-) \\
Fig|7719.3.peg.1819 & 94790 & 77229 & 17562 & - & Protein kinase C, beta type (EC 2.7.II.I) \\
\hline
\end{tabular}

Note: Line corresponded to a gene for SBDS protein ortholog is highlighted. 
Table 2 Tabular display of the chromosomal neighborhood of sbds gene in Plasmodium falciparum genome (SEED db IDs)

\begin{tabular}{llllll}
\hline Fig|36329.I.peg.1979 & 427190 & 428587 & 1398 & + & Eukaryotic translation initiation factor 2 $\gamma$ subunit \\
Fig|36329.I.peg.1980 & 430860 & 429689 & 1005 & - & Hypothetical protein \\
Fig|36329.I.peg.198I & 433950 & 435317 & 519 & + & Hypothetical protein \\
Fig|36329.I.peg.1982 & 437223 & 439465 & 1917 & + & Shwachman-Bodian-Diamond syndrome protein \\
Fig|36329.I.peg.1983 & 440540 & 447009 & 5856 & + & Hypothetical protein \\
Fig|36329.I.peg.1986 & 458795 & 460450 & 1656 & + & Hypothetical protein \\
Fig|36329.I.peg.1987 & 467450 & 461400 & 6051 & - & DNA polymerase I (EC 2.7.7.7) \\
Fig|36329.I.peg.1988 & 468987 & 472015 & 2832 & + & Hypothetical protein \\
Fig|36329.I.peg.1989 & 472942 & 474825 & 1884 & + & GTP-binding protein, putative \\
Fig|36329.I.peg.1990 & 475248 & 477296 & 2049 & + & Putative ribosomal RNA small subunit methyltransferase J (EC 2.I.I.-) \\
\hline
\end{tabular}

Note: Line corresponded to a gene for SBDS protein ortholog is highlighted.

connection for SBDS (Figure 3), with particular TYW1 and SBDS functional coupling as characteristic of all the studied vertebrate genomes.

Wybutosine is a hypermodified guanosine with a tricyclic base found at the $3^{\prime}$-position adjacent to the anticodon of eukaryotic phenylalanine tRNA. The UUU phenylalanine codon is highly prone to frameshift in the $3^{\prime}$ (rightward) direction at pyrimidine $3^{\prime}$ contexts, ${ }^{19}$ and wybutosine supports reading frame maintenance by stabilizing codon-anticodon interactions during decoding on the ribosome. ${ }^{20,21}$ Wybutosine synthesis might proceed through sequential reactions in a multiple protein complex assembled with the precursor tRNA and may be linked to exosomal/proteosomal structure that includes the SBDS protein. Comparative analysis of archeal $s b d s$ loci stresses the potential involvement of SBDS in the exosomal complex where functions of translation, RNA processing, and degradation are tightly coupled.2

The SBDS protein has a thioredoxin fold, which might be directly coupled to the function of wybutosine reductase. Not all stages of wybutosine biosynthesis are known. There is some ambiguity surrounding the source of the $\mathrm{C} 2$ atom in wybutosine, suggested to originate from an intermediate in lysine metabolism. Interestingly, SBDS was shown to interact physically (Reactome $\mathrm{db}$ ) with methylmalonate semialdehyde dehydrogenase, the enzyme in the branched-chain amino acid degradation pathway. It would be too speculative to go further, but the possible involvement of the SBDS protein in biosynthesis of wybutosine merits experimental validation.

Considering an alternative link between wybutosine and SBDS, we may suggest involvement of SBDS in degradation of noncharged or not properly modified tRNA. The tRNA surveillance pathway has been shown to exist in yeast and requires the exosome for polyadenylation and degradation of hypomodified pre-tRNA(i)(Met). ${ }^{23}$ Analogously, tRNA(Phe) without a modified wybutosine residue may be subject to degradation, and therefore SBDS may play some role in this process. The $\mathrm{N}$-terminus of the Yhr087wp yeast protein has a fold similar to that in SBDS. It also has $2.4 \mathrm{e}-06$ and $100.00 \%$ homology with bacterial tRNA pseudouridine 13 synthase, that may support an involvement of Yhr087wp itself and SBDS protein in tRNA modification. Genes do not cluster in $S$. cerevisiae orthologs of SBDS (Q07953, SDO1) and TYW1 (Q08960, Tyw1p). However, the tyw1 locus in $S$. cerevisiae also contains a gene for tRNA pseudouridine synthase 1 (YPL212C) and a number of genes encoding proteins of ribosomal biogenesis.

Establishing a link between SBDS and tRNA modification provides an interesting aspect of crosstalk between cytoskeleton function and translation machinery that may explain a faulty chemotactic phenotype of SBDS malfunction.

Table 3 Tabular display of the chromosomal neighborhood of SBDS-encoding genes in Schizosaccharomyces pombe genome (SEED db IDs)

\begin{tabular}{|c|c|c|c|c|}
\hline Fig |4896. I.peg.3836 & 2660945 & 2660505 & - & SSU ribosomal protein SI2p (S23e), mitochondrial \\
\hline Fig |4896. I.peg.3837 & 2661984 & 2662340 & + & LSU ribosomal protein L40 mt, mitochondrial \\
\hline Fig |4896. I.peg.3838 & 2663620 & 2662700 & - & RNA processing factor I \\
\hline Fig |4896. I.peg.3839 & 2665276 & 2664342 & - & Shwachman-Bodian-Diamond syndrome protein \\
\hline Fig |4896. I.peg.3840 & 2666151 & 2666990 & + & LSU ribosomal protein L24p (L26e), mitochondrial \\
\hline Fig |4896. I.peg.384 I & 2667934 & 2667270 & - & Putative breast adenocarcinoma marker \\
\hline Fig |4896. I.peg.3842 & 2669311 & 2670276 & + & Putative mRNA capping enzyme subunit \\
\hline Fig |4896. I.peg.3843 & $267 \mid 220$ & 2670798 & - & Deoxyuridine 5 and 39;-triphosphate nucleotidohydrolase (EC 3.6.I.23) \\
\hline Fig |4896. I.peg.3844 & 2673717 & 2671936 & - & Mitosis inducer protein kinase cdrl \\
\hline
\end{tabular}

Note: Line corresponded to a gene for SBDS protein ortholog is highlighted. 


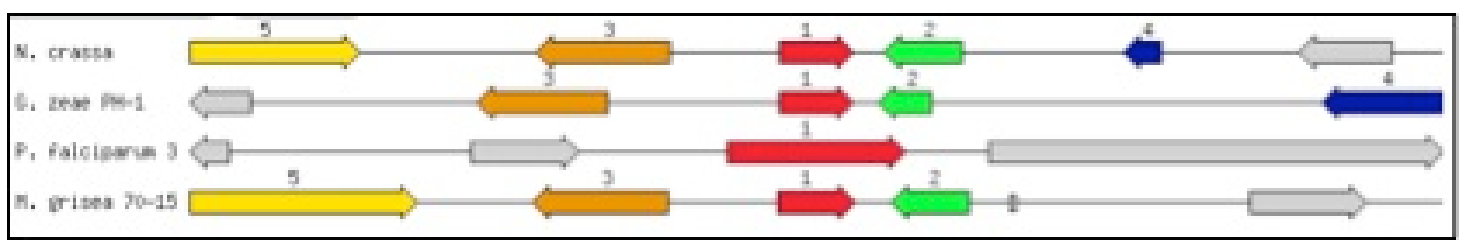

Figure 3 Graphical display of the chromosomal neighborhood of sbds genes in fungal genomes. Arrows correspond to open reading frames encoding: 2- SSU ribosomal protein SI8e (SI3p), 3-carboxypeptidase precursor, and 4- and 5-hypothetical proteins. $100 \mathrm{kbp}$ regions are shown. Organisms are Neurospora crassa, Gibberella zeae, Plasmodium falciparum, and Magnaporthe grisea.

In the P. falciparum and C. intestinalis genomes, SBDS genes are colocalized with functions associated with tRNAregulated components of translational initiation complexes. ${ }^{24}$ GCN1 is a translational activator and regulator of Gcn2p kinase activity. It forms a complex with Gen20p and is proposed to stimulate Gen2p activation by an uncharged tRNA. ${ }^{24,25}$ The second gene colocalized with $s b d s$ in the sea squid genome is a gene for tRNA/RNA cytosine C5 methylase, which is required for initiating tRNA(i) (Met) modification. Eukaryotic and archeal initiation factors 2 are heterotrimeric proteins where only the $\gamma$ subunit of the aIF $2 \alpha \beta$ heterodimer contacts tRNA. ${ }^{26}$ Intriguingly, the $a I F 2 \beta$ gene is colocalized with $s b d s$ in a genome of Plasmodium (Figure 3).

Indirect evidence implicates actin as a cofactor in eukaryotic protein synthesis. The principle function of EF- $1 \alpha$ is to bind aminoacyl-tRNA to the ribosome. EF- $1 \alpha$ also interacts with the cytoskeleton by binding and bundling actin filaments and microtubules, and can alter the assembly of F-actin, a filamentous scaffold on which nonmembrane-associated protein translation take place ${ }^{27}$ (Figure 4). F-actin and aa-tRNA compete for EF-1 $\alpha$, and

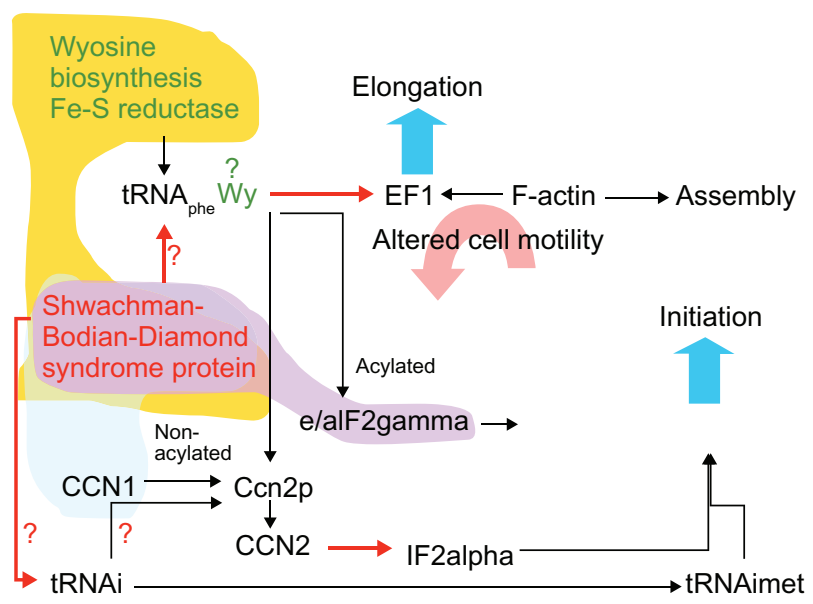

Figure 4 Hypothetical functions of SBDS protein, and their effect on chemotaxis. Clouds represent gene proximity in different groups of organisms, ie, vertebrates (yellow), Ciona intestinalis (blue), and Plasmodium falciparum (purple). their binding is $\mathrm{pH}$-dependent and mutually exclusive. Release of EF- $1 \alpha$ from actin binding was suggested to cause a transient increase in local concentration of the factor to facilitate polypeptide elongation. This interrelationship may ensure that cell proliferation and steady-state protein synthesis is separated from cell migration caused in primitive eukaryotic ancestors by starvation or by an avoidance response to other stressors.

There is also a crosstalk between the two systems in establishing cell polarity during chemotaxis. It has been proposed that the EF-1 $\alpha$-F-actin complex is an important scaffold for anchoring of $\beta$-actin mRNA to sites of active actin polymerization. ${ }^{28}$ Translation only occurs when the RNA-protein complex reaches its destination at the periphery of the cell. ${ }^{29}$ Nothing is known about localization of exosome complexes to cellular lamella, but the processes of translation and degradation of RNA species are likely to be colocalized and to be coordinated by cell locomotion.

It can be suggested that binding of tRNAphe with or without wybutosine modification differentially affects EF- $1 \alpha$. For example, tRNAphe without wybutosine modification can bind to EF- $1 \alpha$ with a higher affinity, and eventually lead to cell polarization/arrest of movement. In this case, the function of the SBDS protein would be in downregulation or destruction of EF-1 $\alpha$, thereby inhibiting tRNA(s). It was shown recently that SBDS protein is also required for release and recycling of the nucleolar shuttling factor, Tif6, from pre-60S ribosomes, ${ }^{30}$ and also for pre-rRNA modifications and final maturation of the ribosome. ${ }^{31}$ Both processes can be regulated and coordinated with ribosomal transport to the cell periphery where actin translation and assembly takes place. ${ }^{32,33}$

We suggest a multiple involvement of SBDS protein in initiation and stability of translation in vertebrates. The suggested roles for this protein in wybutosine/tRNA metabolism would complement its potential involvement in the ribosome assembly recently reported for yeast and would crosstalk tightly, with establishment of cell polarity 
and cell locomotion. Experimental validation of the relationship between SBDS, wybutosine synthesis, and/or degradation of tRNAphe seems plausible, and we hope that our suggestions will attract the attention of biologists in related fields.

It is still not clear what functional features of a gene pair (structural or functional specificity of the encoded proteins, topology of their interaction, presence of a direct proteinprotein contact) correlate significantly with their colocalization. Validation of genomic clustering of genes encoding metabolic functions demonstrates $90 \%$ correlation for yeast and just slightly less for a human genome. A high correlation was also shown between gene colocalization and their temporal and spatial expression profiles. ${ }^{14,16}$ All the existing studies point to phenotypic associations between genes clustered in genomes, but more information is required for a proper large-scale statistical analysis of the correlation. We hope that case-by-case analysis will support the general validity of this method and lead to a routine automatic approach to functional classification of eukaryotic proteins via systematic comparative genomics.

\section{Acknowledgments}

The author thanks Dr Bahram Ibrahimi for his useful comments and also colleagues at the Fellowship of Interpretation of Genomes for their constant support and inspiration.

\section{Disclosure}

The author reports no conflict of interest in this work.

\section{References}

1. Shammas C, Menne TF, Hilcenko C, et al. Structural and mutational analysis of the SBDS protein family. Insight into the leukemiaassociated Shwachman-Diamond syndrome. J Biol Chem. 2005;280: 19221-19229.

2. Bateman A, Birney E, Cerruti, et al. The Pfam Protein Families Database Nucleic Acids Res. 2002;30:276-280.

3. Boocock GR, Morrison JA, Popovic M, et al. Mutations in SBDS are associated with Shwachman-Diamond syndrome. Nat Genet. 2003;33:97-101.

4. de Oliveira JF, Sforça ML, Blumenschein TM, et al. Structure, dynamics, and RNA interaction analysis of the human SBDS protein. $J$ Mol Biol. 2010;396:1053-1069.

5. Wu LF, Hughes TR, Davierwala AP, Robinson MD, Stoughton R, Altschuler SJ. Large-scale prediction of Saccharomyces cerevisiae gene function using overlapping transcriptional clusters. Nat Genet. 2002; 31:255-265.

6. Peng WT, Robinson MD, Mnaimneh S, et al. A panoramic view of yeast noncoding RNA processing. Cell. 2003;113:919-933.

7. Savchenko A, Krogan N, Cort JR, et al. The Shwachman-BodianDiamond syndrome protein family is involved in RNA metabolism. J Biol Chem. 2005;280:19213-19220.
8. Jorgensen R, Ortiz PA, Carr-Schmid A, Nissen P, Kinzy TG, Andersen GR. Two crystal structures demonstrate large conformational changes in the eukaryotic ribosomal translocase. Nat Struct Biol. 2003;10: 379-385.

9. Oubridge C, Ito N, Evans PR, Teo CH, Nagai K. Crystal structure at 1.92 A resolution of the RNA-binding domain of the U1A. Nature. 1994;372:432-438.

10. Stepanovic V, Wessels D, Goldman FD, Geiger J, Soll DR. The chemotaxis defect of Shwachman-Diamond syndrome leukocytes. Cell Motil Cytoskeleton. 2004;57:158-174.

11. Wessels D, Srikantha T, Yi S, Kuhl S, Aravind L, Soll DR. The Shwachman-Bodian-Diamond syndrome gene encodes an RNA-binding protein that localizes to the pseudopod of Dictyostelium amoebae during chemotaxis. J Cell Sci. 2006;119:370-379.

12. R Overbeek R, Fonstein M, D'Souza M, Pusch GD, Maltsev N. The use of gene clusters to infer functional coupling. Proc Natl Acad Sci US A. 1999;96:2896-2901.

13. Vasieva $\mathrm{O}$, Wolf R. Unraveling functional networks: does gene clustering have a meaning? BMC Sys Biol. 2007;1(Suppl v1):84.

14. Lee JM, Sonnhammer EL. Genomic gene clustering analysis of pathways in eukaryotes. Genome Res. 2003;5:875-882.

15. Volpi EV, Chevret E, Jones T, et al. Large-scale chromatin organization of the major histocompatibility complex and other regions of human chromosome 6 and its response to interferon in interphase nuclei. $J$ Cell Sci. 2000;113:1565-1576.

16. Hurst LD, Pal C, Lercher M. The evolutionary dynamics of eukaryotic gene order. Nat Rev Genet. 2004;5:299-310.

17. Vasieva O. The many faces of glutathione transferase pi. Curr Mol Med. 2011;11:129-139.

18. Overbeek R, Begley T, Butler RM, et al. The subsystems approach to genome annotation and its use in the project to annotate 1000 genomes. Nucleic Acids Res. 2005;33:5691-5702.

19. SchwartzR, Curran JF. Analyses of frameshifting at UUU-pyrimidine sites. Nucleic Acids Res. 1997;25:2005-2011.

20. Noma A, Kirino Y, Ikeuchi Y, Suzuki T. Biosynthesis of wybutosine, a hyper-modified nucleoside in eukaryotic phenylalanine tRNA EMBO J. 2006;25:2142-2154.

21. Salomon R, Giveon D, Kimhi Y, Littauer UZ. Abundance of tRNA ${ }^{\text {Phe }}$ lacking the peroxy Y-base in mouse neuroblastoma. Biochemistry. 1976;15:5258-5262.

22. Koonin EV, Wolf YI, Aravind L. Prediction of the archaeal exosome and its connections with the proteasome and the translation and transcription machineries by a comparative-genomic approach. Genome Res. 2001;11:240-252.

23. Kadaba S, Krueger A, Trice T, Krecic AM, Hinnebusch AG, Anderson J. Nuclear surveillance and degradation of hypomodified initiator tRNAmet in S. cerevisiae. Genes Dev. 2004;18:1227-1240.

24. Olsen DS, Savner EM, Mathew A, et al. Domains of eIF1 A that mediate binding to eIF2, eIF3 and eIF5B and promote ternary complex recruitment in vivo. EMBO J. 2003;22:193-204.

25. Kubota H, Ota K, Sakaki Y, Ito T. Budding yeast GCN1 binds the GI domain to activate the eIF2alpha kinase GCN2. J Biol Chem. 2001;276:17591-17596.

26. Yatime L, Mechulam Y, Blanquet S, Schmitt E. Structural switch of the $\gamma$ subunit in an archaeal aIF2 $\alpha \gamma$ heterodimer. Structure. 2006; 14:119-128.

27. Liu G, Tang J, Edmonds BT, Murray J, Levin S, Condeelis J. F-actin sequesters elongation factor from interaction with aminoacyl-tRNA in a pH-dependent reaction. J Cell Biol. 1996;135:953-963.

28. Liu G, Grant WM, Persky D, Latham VM Jr, Singer RH, Condeelis J. Interactions of elongation factor 1a with $\mathrm{F}$-actin and b-actin mRNA: Implications for anchoring mRNA in cell protrusions. Mol Biol Cell. 2002;13:579-592.

29. Hüttelmaier S, Zenklusen D, Lederer M, et al. ZBP1 enhances cell polarity and reduces chemotaxis. $J$ Cell Sci. 2005;120:3173-3178. 
30. Menne TF, Goyenechea B, Sánchez-Puig N, et al. The ShwachmanBodian-Diamond syndrome protein mediates translational activation of ribosomes in yeast. Nat Genet. 2007;39:486-495.

31. Savchenko A, Krogan N, Cort JR, et al. The Shwachman-BodianDiamond syndrome protein family is involved in RNA metabolism. J Biol Chem. 2005;280:19213-19220.
32. Mirra SS, Miles ML, Jacobs J. The coexistence of ribosome-lamella complex and annulate lamellae in chronic lymphocytic leukemia. Ultrastruct Pathol. 1981;2:249-256.

33. Sigler E, Shvidel L, Shtalrid M, Berribi A, Shaft D, Resnitzky P. Ribosome-lamella complexes in a patient with aggressive chronic lymphocytic leukemia. Leuk Lymphoma. 2007;48:434-436.

\section{Publish your work in this journal}

Advances and Applications in Bioinformatics and Chemistry is an international, peer-reviewed open-access journal that publishes articles in the following fields: Computational biomodelling; Bioinformatics; Computational genomics; Molecular modelling; Protein structure modelling and structural genomics; Systems Biology; Computational
Biochemistry; Computational Biophysics; Chemoinformatics and Drug Design; In silico ADME/Tox prediction. The manuscript management system is completely online and includes a very quick and fair peerreview system, which is all easy to use. Visit http://www.dovepress.com/ testimonials.php to read real quotes from published authors.

Submit your manuscript here: http://www.dovepress.com/advances-and-applications-in-bioinformatics-and-chemistry-journal 